\title{
Occupational Stress and Physical Symptoms Original Occupational Stress and Physical Symptoms $\quad$ Article among Family Medicine Residents
}

\author{
So-Myung Choi, Yong Soon Park', Jun-Hyun Yoo*, Go-Young Kim
}

Department of Family Medicine, Samsung Medical Center, Sungkyunkwan University School of Medicine, Seoul; ${ }^{1}$ Health Screening Center, Kangbuk Samsung Hospital, Sungkyunkwan University School of Medicine, Suwon, Korea

Background: The purpose of this study was to examine the levels of occupational stress and physical symptoms among family medicine residents and investigate the effect of subscales of occupational stress on physical symptoms.

Methods: A self-administered questionnaire survey of 1,152 family medicine residents was carried out via e-mail from April 2010 to July 2010. The response rate was $13.1 \%$ and the R (ver. 2.9.1) was used for the analysis of completed data obtained from 150 subjects. The questionnaire included demographic factors, resident training related factors, 24 -items of the Korean Occupational Stress Scales and Korean Versions of the Wahler Physical Symptom Inventory.

Results: The total score of occupational stress of family medicine residents was relatively low compared to that of average workers. The scores of 'high job demand', 'inadequate social support', 'organizational injustice', and 'discomfort in occupational climate' were within the top 50\%. Parameters associated with higher occupational stress included level of training, on-duty time, daily patient load, critical patient assigned, total working days, night duty day, sleep duration, and sleep quality. The six subscales of occupational stress, except for 'Job insecurity', had a significant positive correlation with physical symptom scores after adjustment had been made for potential confounders (total score, $\mathrm{r}=0.325$ and $\mathrm{P}<0.001$; high job demand, $\mathrm{r}=0.439$ and $\mathrm{P}<0.001$ ).

Conclusion: After the adjustment had been made for potential confounders, the total score of occupational stress and six subscales in family medicine residents showed a significant positive correlation with physical symptom scores.

Keywords: Stress; Signs and Symptoms; Family Physicians; Internship and Residency; Questionnaires

\section{INTRODUCTION}

Physicians deal with a patient's life, death and the process of death. In addition, they may work with the calling consciousness. Nevertheless, they may often be exposed to stressful situations. A Received: August 26, 2010, Accepted: November 27, 2012

${ }^{*}$ Corresponding Author: Jun-Hyun Yoo

Tel: +82-2-3410-2449, Fax: +82-2-3410-2459

E-mail: drjohn.yoo@samsung.com

Korean Journal of Family Medicine

Copyright (C) 2013 The Korean Academy of Family Medicine

() This is an open-access article distributed under the terms of the Creative Commons Attribution Non-Commercial License (http://creativecommons.org/licenses/by-nc/3.0) which permits unrestricted noncommercial use, distribution, and reproduction in any medium, provided the original work is properly cited. so-called 'stress' denotes a biological, psychological, or behavioral reaction that occurs in the body in response to mental and physical stimuli impinging on a living body. A proper amount of work stress improves job motivation and productivity. On the contrary, excessive work stress can cause reduction in workrelated desire and productivity, as well as more accidents, and may act as a primary factor for various health disorders including cardiovascular, mental, and musculoskeletal diseases. ${ }^{1)}$ They are subject to great responsibility and clinicians possess significant social prestige and high economic status for their expert knowledge and training. However oppressive stress due to the urgent nature of clinical practice and difficulty of decision making as opposed to those in other occupations. ${ }^{2)}$ Particularly, excessive workload, discord with patients and their guardians, and lawsuits following malpractice are well known as primary stress factors for 
doctors, particularly residents. ${ }^{3,4)}$

A certain level of stress is unavoidable for residents during the residency program and may even be helpful in the performance of a clinician role. On the other hand, undue stress may cause burnout. 'Burnout' is a pathologic symptom, first defined by Maslach et al., which implicates emotional exhaustion and loss of integrity and sensibility caused by tremendous exhaustion or stress. Such burnout negatively affects not only the immediate and long-term physical and mental health of residents, but also threatens patients themselves. ${ }^{6}$ In fact, one foreign study reported that the greater the stress of resident doctors and the lower the sympathy toward stress, the more numerous the malpractice had occurred. ${ }^{7)}$ For this reason, arguments on the issue of a doctor's stress and burnout, as well as the measures for resolving these problems are notably examined in advanced nations. However, unlike efforts seen in such advanced nations, not even assessments on the extent of stress or related factors associated with a doctor's work are performed in Korea. Accordingly, it is imperative to develop tools to grasp the extent of work-related stress and associated factors in Korean doctors. Also, it is urgent to attain the measures of improvement on the basis of this understanding

Family medicine (FM) residents perform outpatient clinical duties in their training program, as well as carry out clinical rotations in other medical specialties to attain expertise in each discipline and execute their responsibilities as the physician in charge in each corresponding department of clinical rotations. Accordingly, FM residents encounter groups of various patients and come across stress diversely in each rotation. Thus, this investigation incorporates a work stress measurement tool for Koreans that has been verified for its validity in various occupations, and examines work-related stress and the level of physical symptoms targeting FM residents engaged in various disciplines. It attempts to carry out discussions by making comparisons between these measured data and standards of the nationwide reference values, and find general characteristics of numerous physical symptoms of FM residents and work stresses associated with these symptoms.

\section{METHODS}

\section{Subjects}

This study included a total of 1,152 nationwide FM residents registered at the Korean Academy of Family Medicine in April 2010. Of these enrollees, there were 411 first year residents, 372 second year residents and 369 third year residents. The data was collected by administering an internet questionnaire via e-mail from April 19, 2010 through July 19, 2010. This study was approved by institutional review board at Samsung Medical Center, Seoul. The purpose of this study was explained to the subjects via e-mail and the questionnaire was administered after obtaining their consent. One hundred fifty-one subjects (survey response rate of $13.1 \%$ ) gave consent for this study and responded to the survey questionnaire. A total of 150 subjects, except for one subject with an inadequate response, were ultimately included in this investigation.

\section{Data Measurement}

The questionnaire consisted of questions assessing general characteristics, job-related stress, and physical symptoms including work-related factors. The general characteristics category contained questions about age, sex, and marital status. The work-related factor category had multiple choice questions about yearly level of residency program, work hours, work days, number of days of a night duty doctor, average number of patients per doctor, and the number of patients per doctor in the intensive care unit. Subjects were asked to fill in the average number of sleeping hours and, with respect to quality of sleep, subjects were invited to select a rating on a four-point scale.

\section{1) Occupational stress}

The Korean Occupational Stress Scales-short form (KOSSSF), ${ }^{1)}$ which had been developed by the Korean Occupational Safety and Health Agency to assess occupational stress of Korean workers, was used. The standard version of KOSS has 8 subcategories in physical environment scale, high job demand, insufficient job control, job insecurity, inadequate social support, organizational injustice, lack of reward, and discomfort in occupational climate, and consists of a total of 43 questions. ${ }^{1)}$ However, the standard version of KOSS has a somewhat larger 
number of questions. Administration of the survey by adding resultant variables other than occupational stress factors, while examining other socio-demographic features and work-related characteristics, has the disadvantage of increasing the volume of questionnaires. ${ }^{1)}$ Thus, this study utilized the KOSS-SF, which had excluded (1) inappropriate questions with respect to internal coherence of questions, validity of reading questions, and factor analysis in the standard version of KOSS, and (2) the area of physical environment with a disposition corresponding to particular occupations. The short version of KOSS is comprised of a total of 24 questions with 7 subcategories that include high job demand (4 questions), insufficient job control (4), job insecurity (2), inadequate social support (3), organizational injustice (4), lack of reward (3) and discomfort in occupational climate (4).

For each question, subjects are asked to respond on a four point scale by choosing one from 'do not agree at all', 'not agree', 'agree', or 'very much agree'. Each response is given a score of 1 , 2, 3, or 4 points. A score of 1, 2, 3, or 4 points, in an increasing order, was allowed for questions with the higher score having the greater stress factor. Analyses were made by re-coding the questionnaire so that a score of $4,3,2$, or 1 , in a decreasing order, was allowed with the higher score having the lower work stress factor. With respect to overall evaluation of occupational stress, this study converted each subcategory so that it would have a total of 100 points, in order to resolve the problem of excessive score reflection of certain subcategories, which could occur when the numbers of questions in 7 subcategories are not the same and the occupational stress factors had to be expressed in a score: converted score for each subcategory $=($ actual score - number of questions) $\times 100 /$ (predicted top score possible - number of questions).

In other words, the actual score of the above equation was substituted with the actual score in each of the 7 subcategories. Each score was added together after converting the score so that a total score would have 100 points. Then, the sum of these scores was divided by 7 (the number of subcategories). And higher scores were associated with higher stress levels. Reliability rate of the questionnaire tool at the time of its development showed the Cronbach $\alpha$ coefficient for each of the subcategories ranged from 0.51 to 0.82 .
2) Physical symptoms

In an attempt to assess the intensity and level of physical symptoms, this study utilized reliability-and-validity-proven Korean Physical Symptom Inventory (K-PSI), ${ }^{8)}$ for which the Wahler Physical Symptom Inventory (WPSI) ${ }^{9)}$ was standardized as the Korean version. K-PSI would have a total of 42 questions including that of headache, fatigue, indigestion, neurologic symptoms, and physical symptoms. Each question had a score scale of six points ranging from 0 point (almost never agree) to 5 points (agree almost everyday) and measured the frequency of manifestation of corresponding symptoms. Regarding the scoring method, the score in each question was added and then the sum was divided by the total number of questions. In the event of omission of any questions, the sum was divided by the number of questions after the omitted ones had been subtracted from the total number of questions. The Cronbach $\alpha$ coefficient was 0.91 at the time of development.

\section{Data Analysis}

The R ver. 2.9.1 (R Foundation for Statistical Computing, Vienna, Austria) was used to make analysis of collected data. Frequency and percentage, mean and standard deviation, or median and range were used to reveal general characteristics and occupational stress, as well as the extent of physical symptoms of subjects. The Mann-Whitney test or Kruskal-Wallis test was used for analysis of occupational stress and to assess the degree of physical symptoms concurring with the general characteristics of subjects. The least significant difference with Bonferroni's correction using ranks was used for post hoc analysis. The correlation between occupational stress and the measure of physical symptoms were examined by using Spearman's correlation analysis. Spearman's partial correlation analysis was incorporated for the assessment of the extent of correlation after relevant variables were compensated for. The level of statistical significance was defined as having a P-value of less than 0.05 .

\section{RESULTS}

\section{General Occupational Characteristics of Family Medicine Residents}

The mean age of 150 subjects was 29.9 years old. Sixty-five 
subjects $(43.3 \%)$ were over the age of 30 years, and 80 subjects (53.3\%) were women. There were 56 first year residents (37.3\%), 52 second year residents (34.7\%), and 42 third year residents (28\%). Mean of on-duty time was 12.5 hours a day and 96

Table 1. Baseline characteristics of study subjects $(n=150)$

\begin{tabular}{|c|c|c|c|}
\hline \multicolumn{2}{|c|}{ Characteristic } & \multirow{2}{*}{$\begin{array}{c}\text { Value } \\
29.9 \pm 3.5\end{array}$} & \multirow{2}{*}{$\begin{array}{c}\text { Median } \\
\text { (range) }\end{array}$} \\
\hline Age (y) & & & \\
\hline & $<30$ & 85 (56.7) & \\
\hline & $\geq 30$ & $65(43.3)$ & \\
\hline \multirow{2}{*}{ Gender } & Male & $70(46.7)$ & \\
\hline & Female & $80(53.3)$ & \\
\hline \multirow[t]{2}{*}{ Marital status } & Single & $85(56.7)$ & \\
\hline & Married & $65(43.3)$ & \\
\hline \multirow[t]{3}{*}{ Level of training } & First grade & $56(37.3)$ & \\
\hline & Second grade & $52(34.7)$ & \\
\hline & Third grade & $42(28.0)$ & \\
\hline \multirow[t]{4}{*}{ On-duty time (h/d) } & & $12.5 \pm 3.0$ & $12(7-24)$ \\
\hline & $<12$ & $54(36.0)$ & \\
\hline & 12 & $45(30.0)$ & \\
\hline & $>12$ & $51(34.0)$ & \\
\hline \multirow{2}{*}{ Total working days (d/wk) } & $\leq 6$ & $109(72.7)$ & \\
\hline & 7 & $41(27.3)$ & \\
\hline \multirow[t]{2}{*}{ Night duty day (d/mo) } & $<10$ & $101(67.3)$ & \\
\hline & $\geq 10$ & 49 (32.7) & \\
\hline \multirow[t]{3}{*}{ Daily patient loads } & $<10$ & $50(33.3)$ & \\
\hline & $10-20$ & $72(48.0)$ & \\
\hline & $>20$ & 28 (18.7) & \\
\hline \multirow[t]{3}{*}{ Critical patients assigned } & & $2.1 \pm 2.5$ & $2(0-10)$ \\
\hline & 0 & $57(38.0)$ & \\
\hline & $\geq 1$ & $93(62.0)$ & \\
\hline \multirow[t]{3}{*}{ Sleep duration (h) } & & $6.0 \pm 0.9$ & $6(4-9)$ \\
\hline & $<6$ & 49 (32.7) & \\
\hline & $\geq 6$ & $101(67.3)$ & \\
\hline \multirow[t]{4}{*}{ Sleep quality } & Very good & $5(3.3)$ & \\
\hline & Good & $70(46.7)$ & \\
\hline & Poor & 55 (36.7) & \\
\hline & Very poor & 20 (13.3) & \\
\hline
\end{tabular}

Values are presented as mean \pm SD or number (\%). subjects (64.0\%) worked 12 hours or more a day in the hospital. Most subjects replied 5 to 6 days for their weekly work days, but 41 subjects $(27.3 \%)$ worked 7 days a week. Forty-nine subjects (32.7\%) worked night duty ten days or more a month. Seventytwo subjects (48\%), the largest number in the study group, were in charge of 10 to 20 patients a day on average at the outpatient department and hospital ward. Twenty-eight subjects (18.7\%) were in charge of more than twenty patients a day. Ninetythree FM residents (62.0\%) were in charge of between one and 10 patients in the intensive care unit (ICU). Mean daily sleep duration was 6 hours and 49 FM residents (32.7\%) had less than 6 hours of sleep a day. In the measurement of quality of sleep on a scale of four points, 75 subjects (50\%) responded as having bad quality of sleep with a score of 0 to 1 point (Table 1 ).

\section{Levels of Occupational Stress and Physical Symptoms}

The mean score for occupational stress was 49.4 points out of a total of 100 points with a distribution minimum of 26.2 points and a maximum of 84.9 points. Looking at the mean scores of 7 subcategories of subordinate factors of occupational stress, the score for high job demand was 50.6 points; insufficient job control, 52.5 points; inadequate social support, 42.8 points; job insecurity, 43.4 points; organizational injustice, 55.0 points; lack of reward, 52.2 points; and discomfort in occupational climate, 49.1 points. The mean score for physical symptoms was 2.1 points (Table 2).

Table 2. Scores of physical symptom and occupational stress

\begin{tabular}{lcl}
\hline \multicolumn{1}{c}{ Variables } & Mean \pm SD & Median (range) \\
\hline Physical symptom scale & $2.1 \pm 0.7$ & \multicolumn{1}{c}{$2.1(1.0-6.0)$} \\
Total score of occupational stress & $49.4 \pm 11.0$ & $48.2(26.2-84.9)$ \\
\multicolumn{1}{c}{ High job demand } & $50.6 \pm 16.3$ & $50.0(0-91.7)$ \\
Insufficient job control & $52.5 \pm 12.7$ & $50.0(16.7-91.7)$ \\
Inadequate social support & $42.8 \pm 14.4$ & $33.3(11.1-77.8)$ \\
Job insecurity & $43.4 \pm 20.7$ & $50.0(0-100)$ \\
Organizational injustice & $55.0 \pm 16.0$ & $50.0(25.0-100)$ \\
Lack of reward & $52.2 \pm 15.2$ & $55.6(22.2-100)$ \\
Discomfort in occupational \\
climate
\end{tabular}




\section{Differences in Occupational Stress and Physical Symptoms According to General Characteristics}

There were no significant differences in both occupational stress and physical symptoms with respect to age, sex and marital status. No significant differences were found in physical symptom scores across the level of training grade, whereas there were significant differences in occupational stress among different yearly levels of training $(\mathrm{P}=0.003)$. The results of post hoc analysis showed significant differences between first year and third year FM residents. Both occupational stress and physical symptoms showed significant differences in the comparisons by dividing mean daily on-duty time into three categories: less than 12 hours, 12 hours, and over 12 hours $(\mathrm{P}<0.001$; $\mathrm{P}=$

Table 3. Occupational stress and physical symptom according to characteristics of subjects

\begin{tabular}{|c|c|c|c|c|c|c|c|}
\hline \multirow{2}{*}{ Variable } & & \multicolumn{3}{|c|}{ Occupational stress } & \multicolumn{3}{|c|}{ Physical symptom } \\
\hline & & Mean \pm SD & Median (range) & $\mathrm{P}$-value & Mean \pm SD & Median (range) & P-value \\
\hline \multirow[t]{2}{*}{ Age (y) } & $<30$ & $49.9 \pm 11.0$ & $48.8(27.0-84.9)$ & 0.421 & $2.0 \pm 0.6$ & $2.1(1.0-3.5)$ & 0.953 \\
\hline & $\geq 30$ & $48.7 \pm 10.9$ & $46.0(26.2-84.1)$ & & $2.1 \pm 0.9$ & $2.0(1.0-6.0)$ & \\
\hline \multirow[t]{2}{*}{ Gender } & Male & $48.9 \pm 11.0$ & $47.2(29.4-84.1)$ & 0.412 & $2.1 \pm 0.9$ & $1.9(1.0-6.0)$ & 0.221 \\
\hline & Female & $49.8 \pm 11.0$ & $48.8(26.2-84.9)$ & & $2.1 \pm 0.6$ & $2.1(1.0-3.6)$ & \\
\hline \multirow[t]{2}{*}{ Marital status } & Single & $50.2 \pm 11.6$ & $49.6(26.2-84.9)$ & 0.254 & $2.1 \pm 0.6$ & $2.1(1.0-3.9)$ & 0.142 \\
\hline & Married & $48.3 \pm 10.0$ & $46.0(33.3-84.1)$ & & $2.0 \pm 0.9$ & $1.9(1.0-6.0)$ & \\
\hline \multirow[t]{3}{*}{ Level of training, grade } & First $^{\mathrm{a}}$ & $51.6 \pm 10.6$ & $50.8(26.2-84.1)$ & 0.003 & $2.2 \pm 0.7$ & $2.2(1.0-4.5)$ & 0.118 \\
\hline & Second $^{\mathrm{b}}$ & $50.4 \pm 12.1$ & $49.4(29.4-84.9)$ & $a>c^{*}$ & $2.0 \pm 0.9$ & $1.9(1.0-6.0)$ & \\
\hline & Third $^{c}$ & $45.2 \pm 8.8$ & 43.1 (33.3-75.4) & & $2.0 \pm 0.7$ & $1.8(1.0-3.9)$ & \\
\hline \multirow[t]{3}{*}{ On-duty time (h/d) } & $<12^{\mathrm{a}}$ & $46.1 \pm 10.0$ & $43.1(31.0-75.4)$ & $<0.001$ & $1.9 \pm 0.6$ & $1.8(1.0-3.4)$ & 0.031 \\
\hline & $12^{\mathrm{b}}$ & $47.6 \pm 10.6$ & $47.2(26.2-67.5)$ & $\mathrm{a}, \mathrm{b}<\mathrm{c}^{*}$ & $2.0 \pm 0.7$ & $1.9(1.0-3.9)$ & $\mathrm{a}<\mathrm{c}^{*}$ \\
\hline & $>12^{\mathrm{c}}$ & $54.5 \pm 10.6$ & $54.8(33.3-84.9)$ & & $2.3 \pm 0.9$ & $2.2(1.0-6.0)$ & \\
\hline \multirow[t]{2}{*}{ Total working days (d/wk) } & $\leq 6$ & $47.9 \pm 10.8$ & $45.6(26.2-84.9)$ & 0.004 & $2.0 \pm 0.7$ & $1.9(1.0-3.9)$ & 0.152 \\
\hline & 7 & $53.2 \pm 10.5$ & $54.8(33.3-84.1)$ & & $2.3 \pm 0.9$ & $2.1(1.0-6.0)$ & \\
\hline \multirow[t]{2}{*}{ Night duty day (d/mo) } & $<10$ & $47.2 \pm 11.3$ & $44.1(26.2-84.9)$ & $<0.001$ & $2.0 \pm 0.7$ & $1.9(1.0-4.5)$ & 0.004 \\
\hline & $\geq 10$ & $53.9 \pm 8.8$ & $55.6(31.0-67.9)$ & & $2.3 \pm 0.8$ & $2.2(1.2-6.0)$ & \\
\hline \multirow[t]{3}{*}{ Daily patient loads } & $<10^{\mathrm{a}}$ & $45.1 \pm 9.5$ & $42.9(27.0-75.4)$ & 0.002 & $1.8 \pm 0.5$ & $1.8(1.0-3.1)$ & 0.010 \\
\hline & $10-20^{\mathrm{b}}$ & $51.6 \pm 11.8$ & $49.8(26.2-84.9)$ & $\mathrm{a}<\mathrm{b}, \mathrm{c}^{*}$ & $2.2 \pm 0.7$ & $2.2(1.0-4.5)$ & $\mathrm{a}<\mathrm{b}^{*}$ \\
\hline & $>20^{\mathrm{c}}$ & $51.2 \pm 9.0$ & $51.2(33.3-67.5)$ & & $2.3 \pm 1.1$ & $2.1(1.0-6.0)$ & \\
\hline \multirow[t]{2}{*}{ Critical patients assigned } & 0 & $44.1 \pm 9.6$ & $42.1(27.0-75.4)$ & $<0.001$ & $1.9 \pm 0.6$ & $1.8(1.0-3.5)$ & 0.011 \\
\hline & $\geq 1$ & $52.6 \pm 10.6$ & $51.2(26.2-84.9)$ & & $2.2 \pm 0.8$ & $2.1(1.0-6.0)$ & \\
\hline \multirow[t]{2}{*}{ Sleep duration (h) } & $<6$ & $52.8 \pm 11.0$ & $50.8(31.0-84.9)$ & 0.005 & $2.2 \pm 0.8$ & $2.1(1.0-4.5)$ & 0.087 \\
\hline & $\geq 6$ & $47.7 \pm 10.6$ & $45.2(26.2-75.4)$ & & $2.0 \pm 0.7$ & $1.9(1.0-6.0)$ & \\
\hline \multirow[t]{2}{*}{ Sleep quality } & Poor & $55.2 \pm 10.0$ & $56.0(33.3-84.9)$ & $<0.001$ & $2.4 \pm 0.7$ & $2.3(1.2-6.0)$ & $<0.001$ \\
\hline & Good & $43.6 \pm 8.5$ & $42.1(26.2-84.1)$ & & $1.8 \pm 0.6$ & $1.7(1.0-4.5)$ & \\
\hline
\end{tabular}

P-value was calculated using Mann-Whitney test or Kruskal-Wallis test.

*LSD (least significant difference) with Bonferroni’s correction using ranks. 
0.031). There were significant differences in occupational stress when mean weekly workdays were divided into two groups: 6 or less days, and 7 days $(\mathrm{P}=0.004)$. Based on 10 days of night duty a month, there were significant differences in the scores of occupational stress and physical symptoms $(\mathrm{P}<0.001$; $\mathrm{P}=$ 0.004). There were significant differences in occupational stress and physical symptoms with respect to the average number of assigned patients or ICU patients cared for by FM residents (all $\mathrm{P}<0.05)$. The scores of occupational stress were significantly higher in subjects with sleep duration of less than 6 hours a day $(P=0.005)$. However, there were no significant differences in physical symptoms $(P=0.087)$. The scores of both occupational stress and physical symptoms were statistically significantly high in subjects with poor quality of sleep (all $\mathrm{P}<0.001$ ) (Table 3 ).

\section{Relationship between Occupational Stress and Physical Symptom Scale}

There was a significant correlation between the total score of occupational stress and physical symptoms $(r=0.553)$. The 6 subscales of occupational stress, except for 'job insecurity', showed a significant positive correlation with physical symptom scores. Even after adjustments of work-related factors of FM residents such as on-duty time, total working days, night duty days, daily patient loads, number of ICU patients assigned, sleep duration, and sleep quality, there was a statistically significant positive correlation between occupational stress and physical symptoms $(r=0.325)$. Even after adjustments of work-related factors for the 6 subscales of occupational stress, except for 'job insecurity', a statistically significant positive correlation was shown between occupational stress and physical symptoms. Among them, stress due to 'high job demand' showed the highest correlation $(r=0.439)$ (Table 4).

\section{DISCUSSION}

The extent of occupational stress perceived by nationwide FM residents showed a mean score of 49.4 points, which belongs to that of the lower $25 \%$ to $50 \%$ group in comparison with the reference values of workers in Korea, corresponding to group with a relatively low occupational stress. Nevertheless, among the subscales, high job demand, insufficient job control, organizational injustice, and discomfort in occupational climate belonged to the top $50 \%$ of nation-wide reference values. The level of occupational stress in these subcategories exceeded the nation-wide mean reference value, entailing a concern on this issue.

The valuation of occupational stress secondary to 'high job demand' means that of the extent of a job burden. Considering the excessive job load and numerous responsibilities demanded upon FM residents, owing to time pressure, work load increase and undue responsibility in the resident training process,

Table 4. Relationship between occupational stress and physical symptom scale

\begin{tabular}{|c|c|c|c|c|}
\hline & \multicolumn{2}{|c|}{ Spearman correlation } & \multicolumn{2}{|c|}{ Partial Spearman correlation } \\
\hline & $\mathrm{r}$ & P-value & $\mathrm{r}$ & P-value \\
\hline Total score of occupational stress & 0.553 & $<0.001$ & 0.325 & $<0.001$ \\
\hline High job demand & 0.546 & $<0.001$ & 0.439 & $<0.001$ \\
\hline Insufficient job control & 0.275 & 0.005 & 0.179 & 0.032 \\
\hline Inadequate social support & 0.311 & $<0.001$ & 0.213 & 0.011 \\
\hline Job insecurity & 0.205 & 0.096 & -0.002 & 0.985 \\
\hline Organizational injustice & 0.434 & $<0.001$ & 0.212 & 0.011 \\
\hline Lack of reward & 0.439 & $<0.001$ & 0.214 & 0.010 \\
\hline Discomfort in occupational climate & 0.551 & $<0.001$ & 0.396 & $<0.001$ \\
\hline
\end{tabular}

Partial Spearman correlation analysis between occupational stress and physical symptom scale was adjusted for on-duty time, total working days, night duty day, daily patient loads, critical patients assigned, sleep duration, and sleep quality.

r: correlation coefficient. 
enormous occupational stress is a matter of course. Particularly, significant differences shown in occupational stress among training yearly levels of FM residents may be associated with the condition in which work load and night duty days are largely concentrated among early year residents. Occupational stress must be enormous in the subscales of 'inadequate social support' and 'organizational injustice', considering the fact that residents are fresh physicians in their early social life and at the lowest level of the vertical social strata among medical doctors. In addition, lack of assistance and support by predecessor doctors, nurses, and other ancillary personnel, organizational discord, and irrational communication in a hospital may work as occupational stress factors. Stress due to 'discomfort in occupational climate' scored 49.1 points, belonging to the group just below the top $75 \%$, which is rather high. Unlike the highly formal, rational job culture of the West, primary stress factors at work in Korea might be related to the group-oriented culture in Korea, system of irrational communication, and unofficial job culture. Summing such factors up, these are stresses which come from collective culture, including factors such as group dining, non-standard or illogical work directions, and authoritarian leadership and hospital hierarchy. It is necessary to bring about change in organization of doctors in hospitals.

Among the subscales of occupational stress in this study, job stress due to 'insufficient job control' belonged to the bottom $25 \%$ to $50 \%$ range, while that of 'job insecurity' and 'lack of reward' belonged to the bottom $25 \%$. This study was the first of its kind to target medical doctors and may not be compared with any previous study. However, the results of this study may reflect the social phenomenon of preference for medical profession in the aspect of expertise and economic compensation.

The mean score of physical symptoms of FM residents was 2.1 points. This score was shown to be higher than that of average healthy adults ( 0.86 points) in the region of Seoul and Gyeonggi Province, diabetes patients (1.22 points), or patients admitted to neuropsychiatric clinic with a chief complaint of physical symptoms (1.87 points), which were measured with the K-PSI used in this study. ${ }^{8)}$ K-PSI was devised to measure the scale and strength of physical symptoms inflicted. Considering the fact that K-PSI has high reliability and validity in the diagnosis of various physical symptoms, it acknowledged distinctive differences in the group of FM residents as opposed to that of a general group. The score of physical symptoms higher than that of diabetes patients might be due to the possibility of having physical manifestations as defensive mechanisms against stress and the actual physical and mental disease that needed to be addressed with an appropriate treatment.

In this study, FM residents most frequently had fatigue, and had complaints of various physical symptoms such as lower back pain, indigestion, myalgia, and headache in a decreasing order. Such results may be explained by the results of a study asserting that continuous stress neuro-physiologically affected the brain, changing pain sensibility leading to physical manifestation and chronic headache, and the outcome of a study reporting that incessant stress was associated with digestive disorders, such as irritable bowel syndrome in the group with immense stress and high score of physical symptoms. ${ }^{10,11)}$ Furthermore, chronic fatigue syndrome is associated with irritable bowel syndrome and fibromyalgia. Pain, digestive disturbance, myalgia, and headache frequently reported by FM residents are included in the diagnostic criteria of chronic fatigue syndrome. Thus, it is necessary in the future to conduct proper evaluations on a correlation between such various physical symptoms reported by FM residents and fatigue of residents. ${ }^{12)}$

The statistical correlation between early-year residents and more on-duty time, work days, night duty days, and patient loads having significantly greater occupational stresses concurred with that of the study conducted on interns, residents, and professors at a US hospital. ${ }^{13)}$ This may be associated with the increased time required for patient care due to less experience seen in doctors of early year residency, and possible discord with senior doctors and senior residents having less on-duty time, work days, night duties, and smaller patient loads. This study showed that the shorter the sleep duration and the worse the quality of sleep had an effect of higher occupational stress. The result of this investigation concurred with that of other studies that a lack of sleep largely affected occupational stress, and inadequate sleep and resultant extreme fatigue might increase malpractice. ${ }^{14)}$

Even after adjustments of on-duty time, work days, night duty days, daily patient loads, number of ICU patients assigned, sleep duration, and sleep quality, there was statistically a significant positive correlation in all 6 subscales, except for work 
insecurity, between occupational stress and physical symptoms. Among them, 'high job demand' showed the highest correlation between work stress and physical symptoms. An intervention in lofty physical symptomatic manifestations associated with occupational stresses may necessitate adjustments in excessive job-related behaviors including the scope of undue on-duty time, night duties, patient loads, and ICU patient loads allotted for residents.

In an attempt to ameliorate various physical symptoms developing among residents, an intervention with respect to management of occupational stress and removal or reduction of causative factors, as well as comprehensive mediation of policy management and personal approach should be undertaken. It was demonstrated in numerous reports, as an issue associated with occupational stress, that there was a correlation between excessive work hours and lack of sleep and job stress and exhaustion with resultant increase in malpractice in the US. A law, enacted in 2003, stipulated that working hours should be 80 hours or less per week and continuous work duration should not exceed 30 hours. ${ }^{15)}$ Furthermore, it was also demonstrated through study that occupational stress and malpractice actually decreased significantly after the enactment of the law. ${ }^{16)}$ Medical discipline related behaviors, which could affect residents and patients, inclusive of continuous work duration and night duties by doctors in their residency program should be managed in our medical community as well. It is urgent to gain a better understanding of occupational stress and the extent of physical symptoms of doctors in their residency program. Measures of mediation such as the Employee Assistance Program for the management of occupational stress, which is widely used in other vocations recently, should be arranged in order to manage job stress and various physical symptoms.

This study had several limitations. The response rate was low as the survey questionnaire was administered via e-mail. The method of verifying precise e-mail delivery of surveying contents to targeted doctors in their FM residency program was inadequate. Despite repeated sending of e-mails pressing for an answer, the response rate was low at $13.1 \%$, which is insufficient to represent all doctors in FM residency programs. Also, medical institutions may have dissimilar FM residency programs and assessment on this aspect has not been included. Nevertheless, the survey was conducted relatively without bias with respect to sex or the year of residency training. The questionnaire was faithfully answered, except for one subject, and inquiries on inclination of the investigated theme were quite meaningful. The reference values of occupational stress among nationwide workers we utilized have implications for a multitude of jobs and include a large number of workers in other age groups. Therefore, comparisons of the precise extent of stress are easier said than done. However, it is quite consequential when examining work stresses of doctors in their residency program, which may interfere with the training process itself. It is also pertinent to suggest modalities for additional management and enhancement in the FM disciplinary process.

A further study is needed for the improvement of occupational stress and physical symptoms of doctors in FM residency program, including those who did not respond to this investigation, and realistic measures should be suggested. Moreover, this study was conducted only among FM residents, but follow-up studies could include doctors of residency programs of other specialties as well as on-field physicians.

\section{CONFLICT OF INTEREST}

No potential conflict of interest relevant to this article was reported.

\section{REFERENCES}

1. Korea Occupational Safety \& Health Agency; Occupational Safety \& Health Research Institute. Standardization of job stress measurement scale for Korean employees (the 2nd year project). Incheon: Korea Occupational Safety \& Health Agency; Occupational Safety \& Health Research Institute; 2004.

2. Freidson E. Professional dominance: the social structure of medical care. New York: Atherton Press; 1970.

3. McCue JD. The effects of stress on physicians and their medical practice. N Engl J Med 1982;306:458-63.

4. Kim YJ, Youn BB. Analysis of professional stress: comparison 
between general practitioners and resident doctors. J Korean Acad Fam Med 1987;8(1):8-17.

5. Maslach C, Shaufeli W, Leiter M, Goldberg J. Job burnout: new directions in research and intervention. Curr Dir Psychol Sci 2003;12:189-92.

6. Thomas NK. Resident burnout. JAMA 2004;292:2880-9.

7. West CP, Huschka MM, Novotny PJ, Sloan JA, Kolars JC, Habermann TM, et al. Association of perceived medical errors with resident distress and empathy: a prospective longitudinal study. JAMA 2006;296:1071-8.

8. Lee BL, Kim DJ, Shin MS, Choi BM. A preliminary study on the standardization of the Korean versions of Wahler Physical Symptom Inventory. J Korean Neuropsychiatr Assoc 2002; 41:146-58.

9. Wahler HJ. Wahler physical symptoms inventory manual. Los Angeles: Western Psychological Services; 1983.

10. Choung RS, Locke GR 3rd, Zinsmeister AR, Schleck CD, Talley NJ. Psychosocial distress and somatic symptoms in community subjects with irritable bowel syndrome: a psychological component is the rule. Am J Gastroenterol
2009; 104:1772-9.

11. Borkum JM. Chronic headaches and the neurobiology of somatization. Curr Pain Headache Rep 2010;14:55-61.

12. Clauw DJ. Perspectives on fatigue from the study of chronic fatigue syndrome and related conditions. PM R 2010;2:41430 .

13. Stucky ER, Dresselhaus TR, Dollarhide A, Shively M, Maynard G, Jain S, et al. Intern to attending: assessing stress among physicians. Acad Med 2009;84:251-7.

14. Papp KK, Stoller EP, Sage P, Aikens JE, Owens J, Avidan A, et al. The effects of sleep loss and fatigue on resident-physicians: a multi-institutional, mixed-method study. Acad Med 2004; 79:394-406.

15. Gopal R, Glasheen JJ, Miyoshi TJ, Prochazka AV. Burnout and internal medicine resident work-hour restrictions. Arch Intern Med 2005; 165:2595-600.

16. Landrigan CP, Rothschild JM, Cronin JW, Kaushal R, Burdick E, Katz JT, et al. Effect of reducing interns' work hours on serious medical errors in intensive care units. $\mathrm{N}$ Engl JMed 2004;351:1838-48. 